\title{
Research Article \\ Gauge and Lorentz Transformation Placed on the Same Foundation
}

\author{
Rein Saar, ${ }^{1}$ Stefan Groote, ${ }^{1,2}$ Hannes Liivat, $^{1}$ and Ilmar Ots ${ }^{1}$ \\ ${ }^{1}$ Loodus- ja Tehnoloogiateaduskond, Füüsika Instituut, Tartu Ülikool, Riia 142, 51014 Tartu, Estonia \\ ${ }^{2}$ Institut für Physik, Johannes-Gutenberg-Universität, Staudinger Weg 7, 55099 Mainz, Germany \\ Correspondence should be addressed to Stefan Groote, groote@thep.physik.uni-mainz.de
}

Received 25 March 2011; Accepted 14 April 2011

Academic Editor: H. Neidhardt

Copyright (C) 2011 Rein Saar et al. This is an open access article distributed under the Creative Commons Attribution License, which permits unrestricted use, distribution, and reproduction in any medium, provided the original work is properly cited.

We show that a "dynamical" interaction for arbitrary spin can be constructed in a straightforward way if gauge and Lorentz transformations are placed on the same foundation. As Lorentz transformations act on space-time coordinates, gauge transformations are applied to the gauge field. Placing these two transformations on the same ground means that all quantized field like spin- $1 / 2$ and spin-3/2 spinors are functions not only of the coordinates but also of the gauge field components. As a consequence, on this stage the (electromagnetic) gauge field has to be considered as classical field. Therefore, standard quantum field theory cannot be applied. Despite this inconvenience, such a common ground is consistent with an old dream of physicists almost a century ago. Our approach, therefore, indicates a straightforward way to realize this dream.

\section{Introduction}

After the formulation of general relativity which explained fources on a geometric ground, physicists and mathematicians tried to incorporate the electromagnetic interaction into this geometric picture. Weyl claimed that the action integral of general relativity is invariant not only under space-time Lorentz transformations but also under the gauge transformation, if this is incorporated consistently [1]. However, the theories at that time were not ready to incorporate this view. Nowadays, we see more clearly that all physical variables (like position, momentum, etc.), quantum wave functions, and fields transform as finitedimensional representations of the Lorentz group. The reason is that interactions between fundamental particles (as irreducible representations of the Poincare group) are most conveniently formulated in terms of field operators (i.e., finite-dimensional representations of the Lorentz group) if the general requirements like covariance, causality, and so forth are to be incorporated in a consistent way. The relation between these two groups and their representations is given by the Lorentz-Poincaré connection [2]. In this paper we show that if gauge transformation is put on the same foundation, the resulting nonminimal "dynamical" 
interaction obeys all necessary symmetries which for higher spins are broken if the interaction is introduced by the usual minimal coupling.

In Section 2 we explain details of the Poincaré group which are necessary in the following. In Section 3 we deal with linear wave equations as objects to the Lorentz transformation. In Section 4 we introduce the external electromagnetic field by a nonsingular transformation. In Section 5 we specify the nonlinear transformation by the claim of gauge invariance of the Poincaré algebra. Finally, in Section 6 we give our conclusions.

\section{The Poincaré Group}

Relativistic field theories are based on the invariance under the Poincare group $p_{1,3}$ (known also as inhomogeneous Lorentz group $\supset \mathcal{\perp}[2-11])$. This group is obtained by combining Lorentz transformations $\Lambda$ and space-time translations $a_{T}$,

$$
(a, \Lambda) \equiv a_{T} \Lambda: \mathbb{E}_{1,3} \ni x^{\mu} \longrightarrow \Lambda_{v}^{\mu} x^{v}+a^{\mu} \in \mathbb{E}_{1,3} .
$$

The group's composition law $\left(a_{1}, \Lambda_{1}\right)\left(a_{2}, \Lambda_{2}\right)=\left(a_{1}+\Lambda_{1} a_{2}, \Lambda_{1} \Lambda_{2}\right)$ generates the semidirect structure of $p_{1,3}$

$$
p_{1,3}=\tau_{1,3} \odot \mathcal{L},
$$

where $\tau_{1,3}$ is the abelian group of space-time translations (i.e., the additive group $\mathbb{R}^{4}$ ) and $\mathcal{L}=\left\{\Lambda: \operatorname{det} \Lambda=+1, \Lambda_{0}^{0} \geq 1\right\}$ is the proper orthochronous Lorentz group acting on the Minkowski space $\mathbb{E}_{1,3}$ with metric

$$
\eta_{\mu \nu}=\operatorname{diag}(1,-1,-1,-1)
$$

The condition of the metric to be invariant under Lorentz transformations $\Lambda$ takes the form

$$
\Lambda_{\rho}^{\mu} \eta_{\mu \nu} \Lambda_{\sigma}^{v}=\eta_{\rho \sigma} .
$$

Under the Lorentz transformation $\Lambda \in \mathcal{L}$ the transformation of the covariant functions $\psi$ according to a representation $\tau(\Lambda)$ of the Lorentz group [3-16] is determined by the commutative diagram

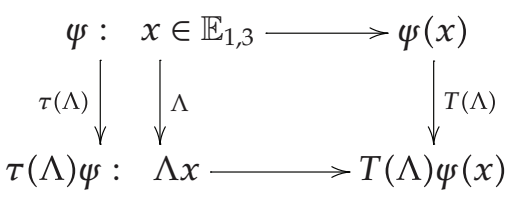

That is,

$$
T(\Lambda) \psi(x)=(\tau(\Lambda) \psi)(\Lambda x) \equiv \psi^{\Lambda}(\Lambda x)
$$

The map $T: \Lambda \rightarrow T(\Lambda)$ is a finite-dimensional representation of $\mathcal{L}$. If we parametrize the element $\Lambda \in \mathcal{L}$ by $\Lambda(\omega)=\exp \left(-(1 / 2) \omega_{\mu \nu} e^{\mu \nu}\right)$ where the Lorentz generators are given by

$$
\left(e_{\mu v}\right)_{\sigma}^{\rho}=-\eta_{\mu}^{\rho} \eta_{v \sigma}+\eta_{\mu \sigma} \eta_{v}^{\rho}
$$


and $\omega^{\mu \nu}=-\omega^{\nu \mu}$ are six independent parameters, the parametrization of $T$ reads

$$
T(\Lambda(\omega))=\exp \left(-\frac{i}{2} \omega_{\mu \nu} s^{\mu \nu}\right) .
$$

The Lorentz group $\mathcal{L}$ is noncompact. As a consequence, all unitary representations are infinite dimensional. In order to avoid this, we introduce the concept of $H$-unitarity (see, e.g., [9] and references therein). A finite representation $T$ is called $H$-unitary if there exists a nonsingular Hermitian matrix $H=H^{\dagger}$ so that

$$
T^{\dagger}(\Lambda) H=H T^{-1}(\Lambda) \Longleftrightarrow s_{\mu \nu}^{\dagger} H=H s_{\mu \nu} .
$$

Notice that an $\mathrm{H}$-unitary metric is always indefinite, so that the inner product $\langle$,$\rangle generated$ by $H$ is sesquilinear sharing the hermiticity condition $\langle\psi, \varphi\rangle=\langle\varphi, \psi\rangle^{*}$. The most famous case of $H$-unitarity is given in the Dirac theory of spin-1/2 particles where $H=\gamma^{0}$.

For an operator $\mathcal{O}[17,18]$ acting on the $\psi$-space of covariant functions (we have to impose the action on covariant functions because in case of higher spins the relations between operators we obtain are valid only as weak conditions) the transformation $\tau(\Lambda)$ in (2.6) is a covariant transformation if the diagram

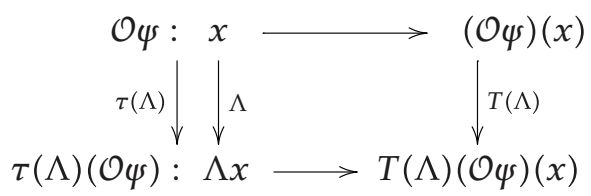

is commutative, that is,

$$
\left(\tau(\Lambda) \mathcal{O} \tau^{-1}(\Lambda)\right)(\Lambda x)(\tau(\Lambda) \psi)(\Lambda x)=T(\Lambda) \mathcal{O}(x) \psi(x) .
$$

Using (2.6) we obtain

$$
\left(\tau(\Lambda) \mathcal{O} \tau^{-1}(\Lambda)\right)(\Lambda x) T(\Lambda) \psi(x)=T(\Lambda) \mathcal{O}(x) \psi(x)
$$

Notice that the covariance of the transformation embodies only the property of equivalence of reference systems. The covariant operator $\mathcal{O}$ is invariant under transformation (2.6) if in addition $\tau(\Lambda) \mathcal{O} \tau^{-1}(\Lambda)=O$. As a consequence we obtain the commutative diagram

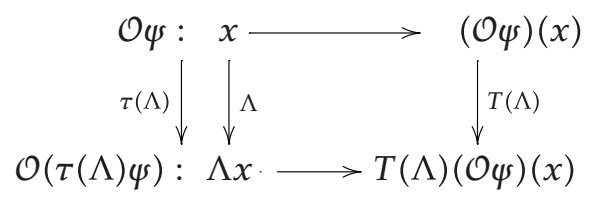

or $\mathcal{O}(\Lambda x) T(\Lambda) \psi(x)=T(\Lambda) \mathcal{O}(x) \psi(x)$ which means

$$
\mathcal{O}(\Lambda x) T(\Lambda)=T(\Lambda) \mathcal{O}(x)
$$


on the $\psi$-space. The invariance is a symmetry of the physical system and implies the conservation of currents. In particular, the symmetry transformations leave the equations of motion form-invariant.

While the Lorentz transformation $T(\Lambda)$ changes the wave function $\psi$ itself as well as the argument of this function (cf. (2.6)), the proper Lorentz transformation $\tau(\Lambda)$ causes a change of the wave function only. On the ground of infinitesimal transformations, this change is performed by the substantial variation. Starting from an arbitrary infinitesimal coordinate transformation $\Lambda(\delta \omega): x^{\mu} \rightarrow x^{\mu}+\delta \omega^{\mu v} x_{v}$, the substantial variation is given by [13]

$$
\delta_{0} \psi(x) \equiv \psi^{\prime}(x)-\psi(x)=-\frac{i}{2} \delta \omega^{\rho \sigma} M_{\rho \sigma} \psi(x)
$$

where $M_{\rho \sigma}=\ell_{\rho \sigma}+s_{\rho \sigma}, \ell_{\rho \sigma}=i\left(x_{\rho} \partial_{\sigma}-x_{\sigma} \partial_{\rho}\right)$. The corresponding finite proper Lorentz transformation can be written as

$$
\tau(\Lambda(\omega))=\exp \left(-\frac{i}{2} \omega_{\mu \nu} M^{\mu v}\right)
$$

and the multiplicative structure of the group generates the adjoint action

$$
\operatorname{Ad}_{\tau(\Lambda)}: M_{\mu \nu} \longrightarrow \tau^{-1}(\Lambda) M_{\mu \nu} \tau(\Lambda)=\Lambda_{\mu}^{\rho} \Lambda_{v}^{\sigma} M_{\rho \sigma} .
$$

Due to (2.9) the generators $s_{\rho \sigma}$ fulfill $s_{\rho \sigma}^{\dagger} H=H s_{\rho \sigma}$. They depend on the spin of the field but not on the coordinates $x_{\mu}$. Therefore, we have $\left[\ell_{\mu \nu}, s_{\rho \sigma}\right]=0$. If a generic element of the translation group is written as

$$
\exp \left(+i a_{\mu} P^{\mu}\right)
$$

the commutator relations of the Lie algebra are given by

$$
\begin{gathered}
{\left[M_{\mu v}, M_{\rho \sigma}\right]=i\left(\eta_{\mu \sigma} M_{\nu \rho}+\eta_{v \rho} M_{\mu \sigma}-\eta_{\mu \rho} M_{v \sigma}-\eta_{v \sigma} M_{\mu \rho}\right)} \\
{\left[M_{\mu v}, P_{\rho}\right]=i\left(\eta_{v \rho} P_{\mu}-\eta_{\mu \rho} P_{v}\right)} \\
{\left[P_{\mu}, P_{v}\right]=0 .}
\end{gathered}
$$

The Casimir operators of the algebra are $P^{2}=P_{\mu} P^{\mu}$ and $W^{2}=W_{\mu} W^{\mu}$, where

$$
W^{\mu}=+\frac{1}{2} \epsilon^{\mu \nu \rho \sigma} M_{v \rho} P_{\sigma}
$$

is the Pauli-Lubanski pseudovector, $\left[P_{\mu}, W_{\nu}\right]=0$. In coordinate representation we have $P_{\mu}=$ $i \partial_{\mu}$, and the finite Poincare transformation has the form

$$
\tau(a, \Lambda): \psi(x) \longrightarrow(\tau(a, \Lambda) \psi)(x)=T(\Lambda) \psi\left(\Lambda^{-1}(x-a)\right)
$$


This relation constitutes the Lorentz-Poincaré connection [2]. While the representation $T$ generally generates a reducible representation of $p_{1,3}$, the spectra of the Casimir operators $P^{2}$ and $W^{2}$ determine the mass and spin content of the system.

\section{The Wave Equations}

As an operator $\mathcal{O}$ in the above sense we consider the operator of the wave equation. The Dirac-type wave equation we will consider has the form

$$
\Phi(\partial) \psi(x) \equiv\left(i \beta^{\mu} \partial_{\mu}-\rho\right) \psi(x)=0,
$$

where $\psi$ is an $N$-component function, $\beta^{\mu}(\mu=0,1,2,3)$, and $\rho$ are $N \times N$ matrices independent of $x$. Following Bhabha's conception [19], it is "...logical to assume that the fundamental equations of the elementary particles must be first-order equations of the form (3.1) and that all properties of the particles must be derivable from these without the use of any further subsidiary conditions."

The principle of relativity states that a change of the reference frame cannot have implications for the motion of the system. This means that (3.1) is invariant under Lorentz transformations. Equivalently, the Lorentz symmetry of the system means the covariance and form-invariance of (3.1) under the transformation in (2.6), that is, the transformed wave equation is equivalent to the old one. Therefore, we require that every solution $\psi^{\Lambda}(\Lambda x)$ of the transformed equation

$$
\Phi^{\Lambda}(\Lambda \partial) \psi^{\Lambda}(\Lambda x)=0
$$

can be obtained as Lorentz transformation of the solution $\psi(x)$ of (3.1) in the original system and that the solutions in the original and transformed systems are in one-to-one correspondence. The explicit form of the covariance follows from (2.11),

$$
\left(\tau(\Lambda) \Phi \tau^{-1}(\Lambda)\right)(\Lambda \partial)(\tau(\Lambda) \psi)(\Lambda x)=T(\Lambda) \Phi(\partial) \psi(x)=0,
$$

and leads to the explicit Lorentz transformations

$$
\beta^{\Lambda \mu}=\Lambda_{\rho}^{\mu} T(\Lambda) \beta^{\rho} T^{-1}(\Lambda), \quad \rho^{\Lambda}=T(\Lambda) \rho T^{-1}(\Lambda) .
$$

The Lorentz invariance is given by the substitution

$$
\Phi(\partial) \psi(x)=0 \stackrel{(2.6)}{\longrightarrow} \Phi(\partial) \psi^{\Lambda}(x)=0 .
$$

or

$$
T^{-1}(\Lambda) \beta^{\mu} T(\Lambda)=\Lambda_{\rho}^{\mu} \beta^{\rho}, \quad T^{-1}(\Lambda) \rho T(\Lambda)=\rho .
$$

The difference of the original and transformed wave equation is given by the wave equation where the wave function $\psi$ is replaced by the substantial variation $\delta_{0} \psi, D(\partial) \delta_{0} \psi(x)=0$. 
As a consequence we obtain $\left[D, M^{\rho \sigma}\right]=0$ or

$$
\left[\beta^{\mu}, s^{\rho \sigma}\right]=i\left(\eta^{\mu \rho} \beta^{\sigma}-\eta^{\mu \sigma} \beta^{\rho}\right), \quad\left[\rho, s^{\rho \sigma}\right]=0
$$

An excellent discussion of such matrices $\beta$ can be found in [13, 19-23]. The hermiticity of the representation $T$ in (2.9) implies the hermiticity of (3.1). Including a still unspecified Hermitian matrix $H$ the hermiticity condition reads $\Phi(\partial)^{\dagger} H \stackrel{!}{=}(\Phi(\partial) H)^{\dagger}=H \Phi(-\partial)$ or

$$
\beta^{\mu \dagger} H=H \beta^{\mu}, \quad \rho H=H \rho .
$$

Writing $\bar{\psi}=\psi^{\dagger} H$, one obtains the adjoint equation

$$
\bar{\psi} \Phi(-\overleftarrow{\partial})=\bar{\psi}\left(-i \beta^{\mu} \overleftarrow{\partial}_{\mu}-\rho\right)=(H \Phi(\partial) \psi)^{\dagger}=0
$$

\section{Introduction of the External Field}

It may be reasonable to introduce an external field directly into the Poincaré algebra which can be applied to classically understand the elementary particle. To do so one has to transform the generators of the Poincare group to be dependent on the external field in such a way that the new, field-dependent generators obey the commutation relations (2.19). As it was proposed by Charkrabarti [24] and Beers and Nickle [25], the simplest way to build such a field-dependent algebra is to introduce the external field $A$ by a nonsingular transformation

$$
\operatorname{Ad}_{\mathcal{V}(A)}: p_{1,3} \longrightarrow p_{1,3}^{d}(A)=\mathcal{V}(A) p_{1,3} \mathcal{V}^{-1}(A)
$$

In case of a particular external electromagnetic field $A$, the external field can be introduced by using an evolution operator $\mathcal{U}(A)$, called the "dynamical" representation [26, 27]. By analogy with the free-particle case one can realize this representation on the solution space of relativistically invariant equations. Expressing the operators explicitly in terms of free-field operators, one obtains the "dynamical" interaction. Applying, for instance, the operator $V(A)$ to $(3.1)$ one obtains

$$
\mho(A): \Phi(\partial) \psi(x)=0 \longrightarrow \Phi^{d}(\partial, A) \Psi(x, A)=0,
$$

where $\Phi^{d}(\partial, A)=\mathcal{U}(A) \Phi(\partial) \mathcal{U}^{-1}(A)$ and

$$
\Psi(x, A)=\mho(A) \psi(x)
$$

(here and in the following we will skip the argument $x$ for $\Psi$ and the argument $\partial$ for $D^{d}$ ). Having introduced the external gauge field $A$, we introduce gauge covariance on the same 
foundation as Lorentz covariance in (2.6), that is, by claiming that the diagram

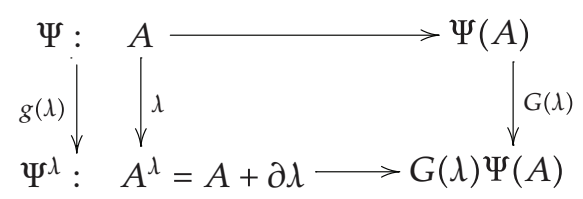

is commutative, that is,

$$
\Psi^{\lambda}(A+\partial \lambda)=G(\lambda) \Psi(A)
$$

According to (2.13), the "dynamical" interaction $\Phi^{d}$ is gauge invariant under the gauge transformation $A \rightarrow A^{\lambda} \equiv A+\partial \curlywedge$ if the diagram

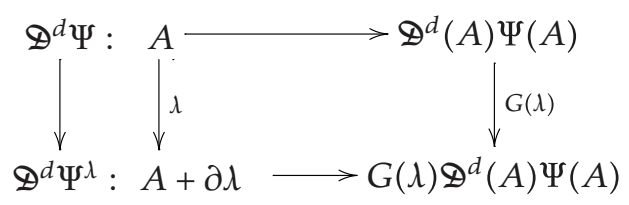

is commutative, that is,

$$
\boldsymbol{\Phi}^{d}(A+\partial \lambda) \Psi^{\lambda}(A+\partial \lambda)=G(\lambda) \Phi^{d}(A) \Psi(A) .
$$

Together with (4.5) we obtain $\Phi^{d}(A+\partial \lambda) G(\lambda) \Psi(A)=G(\lambda) \Phi^{d}(A) \Psi(A)$ or

$$
\Phi^{d}(A+\partial \lambda) G(\lambda)=G(\lambda) \Phi^{d}(A)
$$

on the $\psi$-space. Note that up to now we have not specified the explicit shape of the finite-dimensional representation $G: \lambda \rightarrow G(\lambda)$ of the gauge group.

\section{Specifying $U(A)$ by Gauge Invariance}

At this point we specify $\mho(A)$ by two claims. Due to gauge symmetry as a fundamental principle the dynamical transformation $\mho$ has to be compatible with the gauge transformation. Therefore, we first claim the gauge invariance in (4.8) not only for the operator $\Phi^{d}$ but for the whole dynamical Poincaré algebra $p_{1,3}^{d}(A)$,

$$
p_{1,3}^{d}(A+\partial \lambda) G(\lambda)=G(\lambda) p_{1,3}^{d}(A) .
$$

By using (4.1) and multiplying by $G(\lambda)^{-1}$ from the right we obtain

$$
\mho(A+\partial \lambda) p_{1,3} \mho^{-1}(A+\partial \lambda)=G(\lambda) \mho(A) p_{1,3}(G(\lambda) \mho(A))^{-1} .
$$


This means that the first claim is fulfilled if

$$
V(A+\partial \lambda)=G(\lambda) V(A)
$$

On the other hand, with (4.3) and (4.5) we obtain

$$
\mho^{\lambda}(A+\partial \lambda) \psi(x)=G(\lambda) \circlearrowright(A) \psi(x)
$$

and, therefore, $\mho^{\lambda}=\mho$ on the $\psi$-space. To summarize, by the first claim the gauge symmetry determines the gauge properties of $\mathcal{U}(A)$ and, therefore, of the interacting field $\Psi(A)$.

The second claim is that the dynamical transformation operator $U(A)$ should be of Lorentz type, that is, for the generators $s_{\mu \nu}$ of the Poincaré algebra $p_{1,3}$ one has

$$
\mho(A) s^{\mu \nu} \mho^{-1}(A)=V_{\rho}^{\mu}(A) \mho_{\sigma}^{\nu}(A) s^{\rho \sigma}
$$

which is a local extension of (2.17). $V(A)=V(x, A)$ is the local Lorentz transformation generated by the external field $A$ and obeying

$$
V_{\mu \rho}(A) V_{\sigma}^{\mu}(A)=V_{\rho \mu}(A) V_{\sigma}^{\mu}(A)=\eta_{\rho \sigma} .
$$

If such a local Lorentz transformation exists, the problem is solved. Therefore, in the following we make the attempt to find explicit realizations of the local Lorentz transformation $V_{\mu \nu}(A)$. It is hard to find the Lorentz transformation $V_{\mu \nu}(A)$ in general. However, as first shown by Taub [28], in the case of a plane-wave field we obtain

$$
V_{\mu v}(A)=\eta_{\mu v}-\frac{q}{k_{P}} G_{\mu \nu}-\frac{q^{2}}{2 k_{P}^{2}} A^{2} k_{\mu} k_{v}
$$

where $q$ is the electric charge of the particle and $G_{\mu \nu}=k_{\mu} A_{v}-k_{v} A_{\mu}$. The plane-wave field $A_{\mu}=A_{\mu}(\xi), \xi=k x$ is characterized by its lightlike propagation vector $k_{\mu}, k^{2}=0$, and its polarization vector $a^{\mu}$ such that $a^{2}=-1$ and $k a=0$. The operator $k_{P} \equiv k_{\mu} P^{\mu}$ commutes with any other and has a special role in the theory. For particles with nonzero mass one has $k_{\mu} P^{\mu} \neq 0$. Therefore, for the plane wave the differential operator $1 / k_{P}$ is local and well defined for the plane-wave solution $\psi_{P}$ of the Klein-Gordon equation. In all other cases, $1 / k_{P}$ is assumed to exist.

Note that the plane-wave solution of the Dirac equation was found more than 70 years ago by Wolkow [29] and extended later on to a field of two beams of electromagnetic radiation $[30,31]$. However, these approaches did not make use of the nonsingular transformation $\mho(A)$. The realization of $\mathcal{U}(A)$ can be achieved by the nonsingular transformation $\mho(A)=$ $\mho_{0}(A) \mho_{s}(A)$, where

$$
\begin{gathered}
\mho_{0}(A)=\exp \left\{-i \int \frac{d \xi}{2 k_{P}}\left(2 q(A P)-q^{2} A^{2}\right)\right\}, \\
\mho_{S}(A)=\exp \left\{-\frac{i q}{2 k_{P}} G_{\mu \nu} S^{\mu \nu}\right\} .
\end{gathered}
$$


It has to be mentioned that the evolution operator $U(A)$ may be chosen to be $H$-unitary according to the representation $T$ in (2.9), that is,

$$
\mho^{\dagger}(A) H=H \mho^{-1}(A)
$$

Considering the nonsingular transformation of Dirac-type wave equation

$$
\mho(A):\left(\beta^{\mu} P_{\mu}-m\right) \psi=0 \longrightarrow\left(\Gamma^{\mu}(A) \Pi_{\mu}(A)-m\right) \Psi(A)=0,
$$

with the help of (5.8) the "dynamical" counterparts to the operator $P_{\mu}=i \partial_{\mu}$ can be calculated to be $\Pi_{\mu}(A)=\mho(A) P_{\mu} \mho^{-1}(A)$,

$$
\begin{gathered}
P_{\mu} \longrightarrow \Pi_{\mu}(A)=P_{\mu}+k_{\mu} \frac{q}{2 k_{P}}\left(q A^{2}-2 A P-\not F\right), \\
P^{2} \longrightarrow \Pi^{2}(A)=(P-q A)^{2}-q \not F
\end{gathered}
$$

$\left(F^{\prime} \equiv S^{\mu \nu} F_{\mu \nu}\right)$ while the "dynamical" counterpart to $\beta^{\mu}$ is given by $\Gamma^{\mu}(A)=\mathcal{V}(A) \beta^{\mu} \mho^{-1}(A)$,

$$
\Gamma^{\mu}(A)=V_{v}^{\mu}(A) \beta^{v}=\beta_{\mu}-\frac{q}{k_{P}}\left(\frac{q}{2 k_{P}} A^{2} k_{\mu} k_{v}+G_{\mu v}\right) \beta^{v}
$$

In terms of $\Pi_{\mu}(A)$ and $\Gamma^{\mu}(A)$ we have

$$
\boldsymbol{\Phi}^{d}(A) \Psi(A)=\left(\Gamma^{\mu}(A) \Pi_{\mu}(A)-m\right) \Psi(A)=0 .
$$

However, expressed in terms of $D_{\mu}=P_{\mu}-q A_{\mu}$ and $\beta^{\mu}$, we obtain

$$
\Phi^{d}(A) \Psi(A) \equiv\left(\beta^{\mu} D_{\mu}-\frac{q}{2 k_{P}} k F^{\prime}-m\right) \Psi(A)=0
$$

where $k \equiv \beta^{\mu} k_{\mu}$. This interaction is nonminimal. However, as we have shown before, it is determined completely by the claim of gauge invariance.

Note that due to the antimutation of the $\gamma$-matrices, in the spin- $1 / 2$ case the dynamical interaction in (5.15) reduces to the minimal coupling. However, in order to obtain the correct values of the gyromagnetic factor, in some cases the (phenomenological) Pauli term $\gamma_{\mu} \gamma_{\nu} F^{\mu \nu}$ has to be added by hand to the minimal coupling of the Dirac equation (see also [32, page $109])$. In case of plane waves the exact solution of this (supplemented) Dirac equation as given by Charkrabarti [24] obeys the same gauge invariance condition $\Psi(A+\partial \lambda)=G(\lambda) \Psi(A)$. This property is found also in the book by Fried [33].

Finally, as a consequence of the explicit form (5.8), the associated transformation of the evolution operator $\mho(A)$ under the local gauge transformation for the plane wave field,

$$
A_{\mu}(\xi) \longrightarrow A_{\mu}(\xi)+\partial_{\mu} \lambda(\xi)
$$


becomes

$$
\mho(A) \longrightarrow \mho(A+\partial \lambda)=e^{-i q \lambda} \mathcal{U}(A)
$$

As an example of higher spin, the spin-3/2 case is considered in detail in [34]. As it turns out, the Rarita-Schwinger spin-3/2 equation on the presence of a "dynamical" interaction is algebraically consistent and causal.

\section{Conclusions}

As a consequence of gauge invariance and Lorentz type of $\mathcal{U}(A)$ we obtain

(1) the invariance of the wave function under gauge transformations,

$$
\Psi^{\lambda}(A+\partial \lambda)=\mho^{\lambda}(A+\partial \lambda) \psi=\mho(A+\partial \lambda) \psi=\Psi(A+\partial \lambda),
$$

that is, $\Psi^{\lambda}=\Psi$,

(2) the explicit shape of $G(\lambda)$ in (4.5),

$$
\Psi^{\lambda}(A+\partial \lambda)=\mathcal{U}(A+\partial \lambda) \psi=e^{-i q \lambda} \mathcal{U}(A) \psi=e^{-i q \lambda} \Psi(A)
$$

that is, $G(\lambda)=e^{-i q \lambda}$,

(3) the invariance of $\Phi^{d}$ under gauge transformations from (4.7) and

$$
\boldsymbol{\Phi}^{d}(A+\partial \lambda) \Psi^{\lambda}(A+\partial \lambda)=\Phi^{d}(A+\partial \lambda) e^{-i q \lambda} \Psi(A)
$$

that is, $\Phi^{d}(A+\partial \lambda) G(\lambda)=G(\lambda) \Phi^{d}(A)$ on the $\psi$-space,

(4) the "dynamical" interaction for any spin as given by

$$
\Phi^{d}(A) \Psi(A)=\left(\beta^{\mu} D_{\mu}-\frac{q}{2 k_{P}} k \not F-m\right) \Psi(A)=0
$$

being nonminimal but completely determined by gauge invariance, thereby causing Poincaré symmetry,

(5) as a consequence of (5.12), the gyromagnetic factor in the presence of a "dynamical" interaction as being $g=2$ for any spin [27].

Let us close again with Weyl In [1] he honestly confessed: "Die entscheidenden Folgerungen in dieser Hinsicht verschanzen sich aber noch hinter einem Wall mathematischer Schwierigkeiten, den ich bislang nicht zu durchbrechen vermag." ("However, the crucial consequences in this respect entrench oneself still behind a bank of mathematical difficulties which up to now I am not able to penetrate.") We hope that our work breaks a small bay into this mathematical bank. 


\section{Acknowledgments}

The work is supported by the Estonian target financed Project no. 0180056s09 and by the Estonian Science Foundation under grant no. 8769. S. Groote acknowledges support by the Deutsche Forschungsgemeinschaft (DFG) under Grant 436 EST 17/1/06.

\section{References}

[1] H. Weyl, "Eine neue Erweiterung der Relativitätstheorie," Annalen der Physik, vol. 364, no. 10, pp. 101-133, 1919.

[2] W.-K. Tung, Group Theory in Physics, World Scientific, Philadelphia, Pa, USA, 1985.

[3] E. Wigner, "On unitary representations of the inhomogeneous Lorentz group," Annals of Mathematics. Second Series, vol. 40, no. 1, pp. 149-204, 1939.

[4] E. Wigner, "On unitary representations of the inhomogeneous Lorentz group," Nuclear Physics B (Proceedings Supplements), vol. 6, pp. 9-64, 1989, (Reprint from Annals of Mathematics, vol. 40, no. 1, pp. 149-204, 1939).

[5] V. Bargmann, "On unitary ray representations of continuous groups," Annals of Mathematics. Second Series, vol. 59, pp. 1-46, 1954.

[6] C. Fronsdal, "Unitary irreducible representations of the Lorentz group," Nuovo Cimento, vol. 113, pp. 1367-1374, 1959.

[7] R. Shaw, "Unitary representations of the inhomogeneous Lorentz group," Nuovo Cimento, vol. 33, pp. 1074-1090, 1964.

[8] H. Joos, "Zur Darstellungstheorie der inhomogenen Lorentzgruppe als Grundlage quantenmechanischer Kinematik," Fortschritte der Physik, vol. 10, no. 3, pp. 65-146, 1962.

[9] U. H. Niederer and L. O'Raifeartaigh, "Realizations of the Unitary Representations of the Inhomogeneous Space-Time Groups I. General Structure," Fortschritte der Physik, vol. 22, no. 3, pp. 111-129, 1974.

[10] Y. Ohnuki, Unitary Representations of the Poincaré Group and Relativistic Wave Equations, World Scientific, Teaneck, NJ, USA, 1988.

[11] Y. S. Kim and M. E. Noz, Theory and Applications of the Poincaré Group, Fundamental Theories of Physics, D. Reidel, Dordrecht, The Netherlands, 1986.

[12] V. Bargmann and E. P. Wigner, "Group theoretical discussion of relativistic wave equations," Proceedings of the National Academy of Sciences of the United States of America, vol. 34, pp. 211-223, 1948.

[13] E. M. Corson, Introduction to Tensors, Spinors, and Relativistic Wave Equations, Blackie and Sons, London, UK, 1953.

[14] D. L. Pursey, “General theory of covariant particle equations," Annals of Physics, vol. 32, pp. 157-191, 1965.

[15] W.-K. Tung, "Relativistic wave equations and field theory for arbitrary spin," Physical Review, vol. 156, no. 5, pp. 1385-1398, 1967.

[16] A. S. Wightman, "Invariant wave equations: general theory and applications to the external field problem," in Invariant wave equations (Proceedings of "Ettore Majorana" International School of Mathematical Physics of Erice, 1977), vol. 73 of Lecture Notes in Phys., pp. 1-101, Springer, Berlin, Germany, 1978.

[17] N. Giovannini, "Covariance group in the presence of external electromagnetic fields," Helvetica Physica Acta, vol. 50, no. 3, pp. 337-348, 1977.

[18] A. Janner and E. Ascher, "Space-time symmetry of transverse electromagnetic plane waves," Helvetica Physica Acta, vol. 43, pp. 296-303, 1970.

[19] H. J. Bhabha, "Relativistic wave equations for the elementary particles," Reviews of Modern Physics, vol. 17, pp. 200-216, 1945.

[20] M. Fierz, “Über die relativistische Theorie kräftefreier Teilchen mit beliebigem Spin,” Helvetica Physica Acta, vol. 12, pp. 3-17, 1939.

[21] E. Wild, "On first order wave equations for elementary particles without subsidiary conditions," Proceedings of the Royal Society. London. Series A, vol. 191, pp. 253-268, 1947.

[22] I. M. Gel'fand, R. A. Minlos, and Z. Y. Shapiro, Representations of Rotation and Lorentz Groups and Their Applications, Pergamon Press, Oxford, UK, 1963.

[23] M. A. Naimark, Linear Representations of the Lorentz Group, Pergamon Press, Oxford, UK, 1964. 
[24] A. Charkrabarti, "Exact solution of the Dirac-Pauli equation for a class of fields: precession of polarization," Il Nuovo Cimento A, vol. 56, no. 3, pp. 604-624, 1968.

[25] B. Beers and H. H. Nickle, "Algebraic solution for a Dirac electron in a plane-wave electromagnetic field," Journal of Mathematical Physics, vol. 13, pp. 1592-1595, 1972.

[26] R. Saar, R. K. Loide, I. Ots, and R. Tammelo, "“Dynamical” representation of the Poincaré algebra for higher-spin fields in interaction with plane waves," Journal of Physics. A, vol. 32, no. 12, pp. 2499-2508, 1999.

[27] I. Ots, R. Saar, R. K. Loide, and H. Liivat, "'Dynamical" non-minimal higher-spin interaction and gyromagnetic ratio $\mathrm{g}=2$," Europhysics Letters, vol. 56, no. 3, pp. 367-371, 2001.

[28] A. H. Taub, "A special method for solving the dirac equations," Reviews of Modern Physics, vol. 21, no. 3, pp. 388-392, 1949.

[29] D. M. Wolkow, “Über eine Klasse von Lösungen der Diracschen Gleichung,” Zeitschrift für Physik, vol. 94, no. 3-4, pp. 250-260, 1935.

[30] N. D. Sen Gupta, "On the solution of the Dirac equation in the field of two beams of electromagnetic radiation," Zeitschrift für Physik, vol. 200, no. 1, pp. 13-19, 1967.

[31] M. Pardy, "Volkov solution for two laser beams and ITER," International Journal of Theoretical Physics, vol. 45, no. 3, pp. 647-659, 2006.

[32] J. J. Sakurai, Modern Quantum Mechanics, Addison-Wesley, Reading, Mass, USA, 1993.

[33] H. M. Fried, Basics of Functional Methods and Eikonal Models, Editions Frontières, Gif-sur-Yvette, France, 1990.

[34] R. Saar, S. Groote, H. Liivat, and I. Ots, "“Dynamical” interactions and gauge invariance," http:// arxiv.org/abs/0908.3761. 


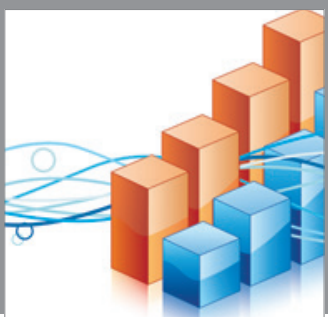

Advances in

Operations Research

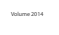

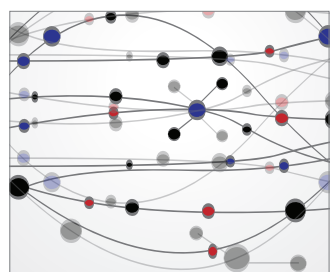

\section{The Scientific} World Journal
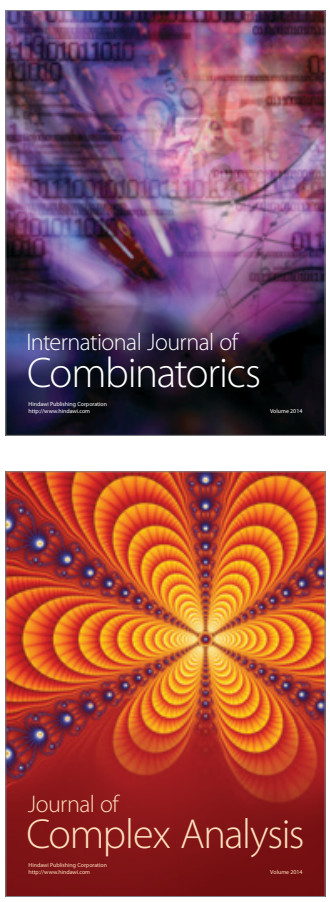

International Journal of

Mathematics and

Mathematical

Sciences
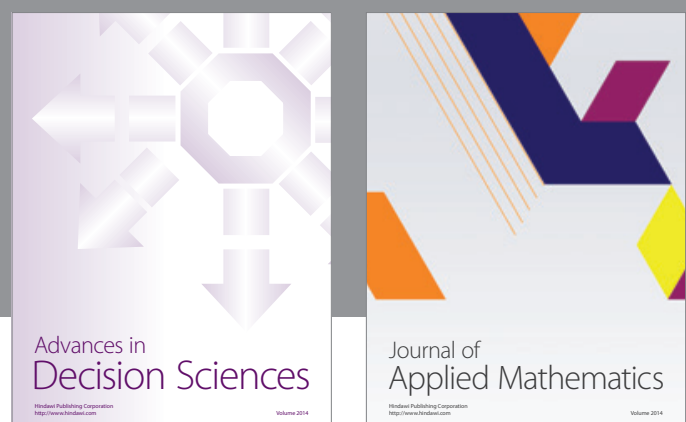

Journal of

Applied Mathematics
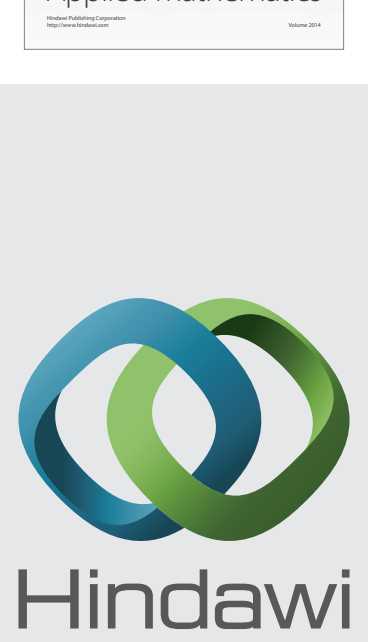

Submit your manuscripts at http://www.hindawi.com
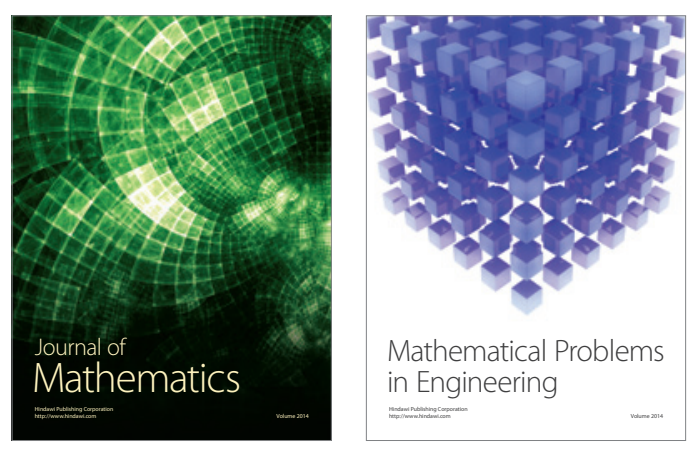

Mathematical Problems in Engineering
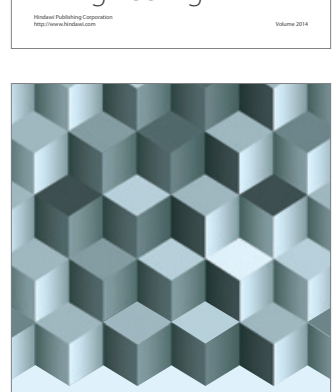

Journal of

Function Spaces
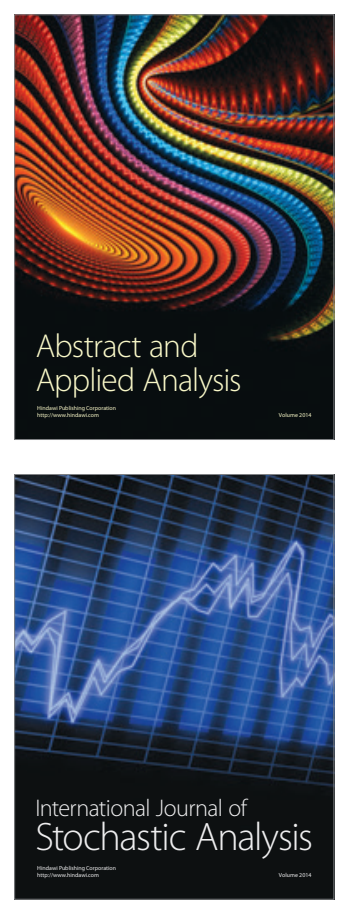

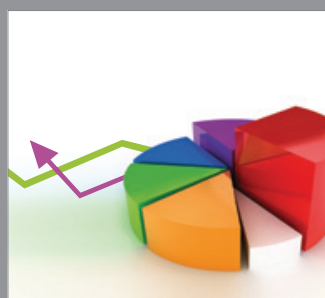

ournal of

Probability and Statistics

Promensencen
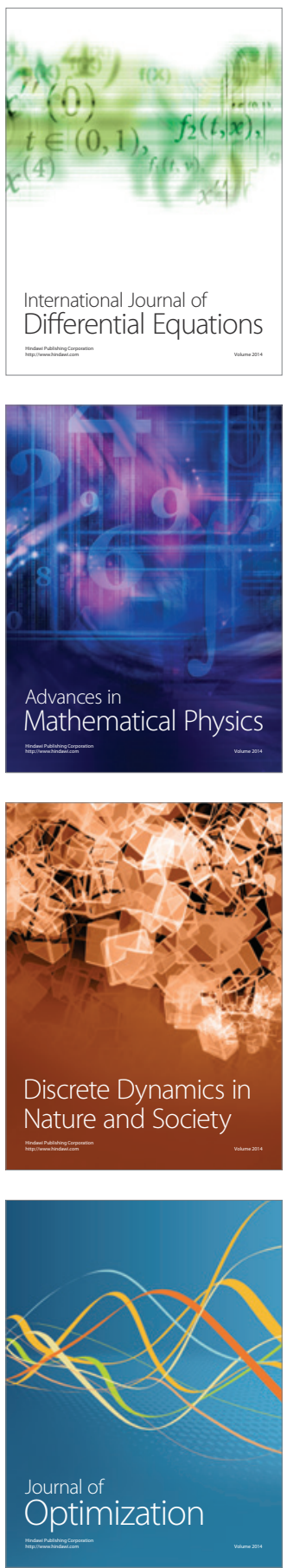